\title{
Interdisciplinary collaboration in reablement - a qualitative study
}

This article was published in the following Dove Press journal:

Journal of Multidisciplinary Healthcare

5 May 2017

Number of times this article has been viewed

\author{
Arvid Birkeland ${ }^{1,2}$ \\ Hanne Tuntland $d^{1,3,4}$ \\ Oddvar Førland 1,5 \\ Frode Fadnes Jakobsen ${ }^{1,4,6}$ \\ Eva Langeland ${ }^{1,4,6}$ \\ 'Centre for Care Research Western \\ Norway, Bergen, ${ }^{2}$ Western Norway \\ University of Applied Sciences, \\ Haugesund, ${ }^{3}$ Faculty of Health \\ and Social Sciences, Department \\ of Occupational Therapy, \\ Physiotherapy and Radiography, \\ ${ }^{4}$ Western Norway University of \\ Applied Sciences, ${ }^{5}$ Faculty of Health \\ Sciences, VID Specialized University, \\ ${ }^{6}$ Faculty of Health and Social \\ Sciences, Department of Nursing, \\ Bergen, Norway
}

Correspondence: Arvid Birkeland Western Norway University of Applied Sciences, Bjornsonsgate 45, 5528 Haugesund, Norway

Email arvid.birkeland@hvl.no
Background: In-depth knowledge regarding interdisciplinary collaboration, a key feature in reablement, is scarce.

Objective: To elucidate how the interdisciplinary collaboration in reablement worked in a Norwegian context.

Sample and methods: Seven focus group interviews were conducted with 33 health care providers working in interdisciplinary reablement teams in seven municipalities across the country. The focus group interviews were transcribed and an hermeneutical analysis was conducted. Results: The analysis resulted in four main themes: "participant's own goals as a common interdisciplinary platform", "a positive professional community", "learning from each other's skills and competencies" and "new roles and joint efforts but specific competencies". The results show that interdisciplinary collaboration in reablement depends on participants defining their own rehabilitation goals, which function as a professional unifying platform for the interdisciplinary collaboration. The challenges for participants in reablement are often complex and include assessments, effort and a need for close collaboration between several different professionals. A tight interdisciplinary collaboration causes major changes in roles, often from a particular role to a more general role with broader job tasks. Although different professionals perform the same rehabilitation tasks, it is important that each professional contributes their unique competence and thus together they complete each other's competencies.

Conclusion: Factors that have a positive impact on interdisciplinary collaboration in reablement are participants' definitions of their goals, number and variety of professionals involved, how closely these professionals collaborate, the amount of time for communication and shared planning and decision making.

Keywords: rehabilitation, reablement teams, home care service, rehabilitation goals, roles

\section{Introduction}

Reablement, also known as "restorative care", is rehabilitation taking place in peoples" own home as part of the home care service. The main purpose of reablement is to improve functions related to the challenges individuals face in their own homes in their everyday lives. Although rehabilitation may be important for individuals in their own homes, rehabilitation has traditionally not been an active part of the home care service. The evidence base concerning the effects of reablement is inconclusive, but promising. ${ }^{1-3}$ Four recent systematic reviews conclude that there is uncertainty regarding the effects of reablement on health and function. ${ }^{4-7}$ However, some qualitative studies indicate that reablement is of great importance for participants and that the 
reablement team supports older adults to regain confidence in performing everyday activities as well as participating in society. ${ }^{8,9}$ Specialized rehabilitation is essentially localized in hospitals or institutions and has often focused on specific diseases, injuries and specific training. To distinguish between reablement and other rehabilitation services Cochrane et al, ${ }^{4}$ have defined reablement as an intensive, time-limited, personcentered and goal-directed intervention that is provided by an interdisciplinary team in the home setting and/or in the local community.

A core factor in reablement is interdisciplinary work, and interdisciplinary teams have been central in the implementation of reablement in different municipalities. ${ }^{4,10-12}$ An interdisciplinary team can be defined as a group of professionals, ideally at the same hierarchical level, working together to achieve common goals. ${ }^{13-15}$ Traditionally interdisciplinary collaboration has been dominated by a thinking and culture that is characterized by autonomy and dominance, rather than trust and collegiality. However some studies show that rehabilitation teams experience teamwork, in general, as positive, exciting and challenging. ${ }^{10-12,16-19}$ There are indications that effective rehabilitation teams are characterized by trust, knowledge, shared responsibility, mutual respect, commitment, optimism and well-being. ${ }^{15,19}$ Communication is a key feature in interdisciplinary collaboration, and some studies describe communication as the "glue" that binds the team together and enables collaboration. ${ }^{15}$ One study particularly highlight the importance of discussions, which often also have a learning effect. ${ }^{18}$

There does not seem to be a generic term or model for interprofessional collaboration, but the most frequent terms are multidisciplinary (synonymous with multiprofessional), interdisciplinary (synonymous with interprofessional or integrative) or transdisciplinary team or collaboration (synonymous with transprofessional). ${ }^{14,19}$ The term "multidisciplinary" often refers to a process where different disciplines work independently, but share information with each other. Focus is the task, rather than the collective working process. Each team member, often with a high degree of expertise, works alone and their contributions can be performed without any input from the others. ${ }^{19}$ Recent reablement studies use the term "integrated multidisciplinary team" when teams operate integrated within the home care service. ${ }^{10}$ The term "interdisciplinary" is characterized by interactive tasks, and implies high levels of communication, mutual planning, shared responsibilities and decisions. Finally, the term "transdisciplinary team" is characterized by an integrative process where team-members operate closely with each other, build consensus, exchange knowledge and skills, and as a result exceed traditional professional boundaries. ${ }^{15,19}$ Teamwork might be described as a continuum from multidisciplinary teams, where professionals are working almost professionally independent, through interdisciplinary teams, and finally to the transdisciplinary model where team-members collaborate most closely with each other. ${ }^{15,19}$ Most studies seem to use the term multidisciplinary or interdisciplinary, but few seem to have a clear awareness of or explicit definition of multi-, inter- or transdisciplinary team. ${ }^{10-13,18,20,21}$ Some studies emphasize that the more signs there are that transdisciplinary team collaboration is developing, the more efficiency and thriving there is in the team. ${ }^{19}$ Knowledge of each other's roles and mutual trust between the professions is a prerequisite for high quality interdisciplinary collaboration. ${ }^{11}$ It can be a challenge to see one's own and others' role in the team, and this is dependent on each profession working in a context where they have the opportunity to demonstrate their professional practice and their identity to the others. ${ }^{15,22}$

Given that there are few studies of employees' experiences of interdisciplinary collaboration in reablement, the purpose of this study was to elucidate how such collaboration worked, and to understand team members' experiences of the relationships between each other and team organization. Although it does not seem to be a common term for collaboration between professionals in rehabilitation teams, we here choose the term interdisciplinary because we are focusing on the relationship and collaboration between the various disciplines within the teams. The following research question was posed: "How do professionals experience the interdisciplinary collaboration within reablement teams?"

\section{Methods}

This study was part of a larger research and evaluation project initiated by the Norwegian Directorate of Health focusing on models and effects of reablement in 43 Norwegian municipalities. The study is based on data obtained from this evaluation. ${ }^{23}$

\section{Research design}

This study had a qualitative approach with an open and emergent design. ${ }^{25}$ It involved collecting data through focus group interviews with selected persons, and was open to what "emerged" through the descriptions and the information collected. A focus group interview is an interview with a limited number of persons where the researcher introduces topics to be discussed and where the group members are free to discuss with and learn from each other. This is a method which has 
been proved useful when the aim is to identify shared experiences and perceptions, including different perceptions. ${ }^{26}$

\section{Data collection and selection}

A total of 33 participants were recruited (nine physiotherapists, nine nurses, seven occupational therapists, four social educators, three auxiliary nurses and one social worker) from seven rehabilitation teams in seven municipalities in six counties across Norway. The number of inhabitants in the municipalities ranged from $\sim 4,000$ to 90,000 . The primary health care service employed and managed the reablement teams. Under this management the seven municipalities had completed reablement with a total of 291 persons, and so had considerable experience with a large number of people in the practice of reablement. Focus group participants were selected and recruited through designated leaders/contact persons in each municipality based on agreed criteria. The participants were selected on the basis that they were professionals, preferably with a bachelor's degree and had previously been selected to work specifically on reablement with other professionals. All the municipalities, in the study, had begun their reablement service with teams consisting of at least three different professionals, usually physiotherapists, nurses and occupational therapists, and these teams had been functioning for between 1 and 2 years. Some municipalities had large teams with $12-15$ employees. Others had only 3 or 4 , but instead of engaging more employees within the team they wanted to cooperate with the home care service.

The number of people in the focus groups ranged from 4 to 6 and the focus group interviews lasted for 1-1.5 hours. The focus group interviews were conducted in the municipalities by the first author as moderator.

Prior to the focus group interviews an interview guide was written by all the authors in collaboration based on the study's aims and purpose. Although it was used as an interview guide, the focus groups gave a lot of information and reflections without being directly asked. The interview guide contained the following main headings:

- Experiences and thoughts on the interdisciplinary collaboration

- Collaboration arenas, meetings, etc

- Collaboration interval

- Roles, changing roles and if so, how

- Work satisfaction - changes and if so, how and why

- Perceptions of how the interdisciplinary work generally works

- Any other topics, views and comments on interdisciplinary collaboration.

\section{Data analysis}

Focus group interviews were recorded using a sound recorder with the moderator transcribing the content verbatim later. By listening to the interviews the first author had the opportunity to capture breaks, laughter, voice and other sounds that may have affected the interpretation. The analysis was based on a hermeneutic approach both in relation to the conducting of the focus group interviews and in relation to the theming of data. In hermeneutics, interpretation and understanding are central concepts, and this study has focused on understanding how participants experienced interprofessional collaboration. Members of the focus groups were working in the context of people's homes, the home care service and within a team of professionals. A main theme in a hermeneutic approach is that the significance of a part can only be understood if it is related to the whole context. ${ }^{27}$ An expanded understanding was, therefore, achieved through a circular process of alternating between a partial and holistic/whole perspective in relation to the team-members' experiences. In the hermeneutic approach, there is also a special relation between preunderstanding and understanding. ${ }^{27} \mathrm{All}$ researchers have a professional preunderstanding based on their scientific knowledge and experiences, commitment and prejudices. These preunderstandings have meaning and importance but researchers must be aware of their preunderstandings in a way that forms a basis for an expanded understanding. ${ }^{27}$ The practical reading and specific analysis was conducted by the first author, but the written text was made in collaboration with the other authors. Throughout the text reading the question addressed was: "how do the professionals experience the interdisciplinary collaboration?" Through this process the different themes were distinguished.

The specific and practical analysis involved the following steps:

- analysis in the interview setting,

- listen to recorded focus group interviews,

- transcribe the verbatim text,

- read through all the focus group interviews to get an overview,

- a new reading, selecting main themes,

- a new reading to identify subthemes,

- transform the data into a whole with main themes as headlines confirmed by written and verbatim examples from informants.

The steps of identifying the main themes and subthemes were time consuming and consisted of several textual readings. 


\section{Ethical considerations}

The study was approved by The Regional Ethical Committee for Medical and Health Research Ethics (REK VWest, 2014/57-1). Study participants were recruited through the contact person in each municipality and the participants were given oral and written information about the research project. Participation in the study was voluntary and participants confirmed willingness to participate and signed a written informed consent form for this study. They were informed that they could withdraw from the study without consequences. In addition, the participants were informed about researchers' confidentiality, including informants' anonymity.

\section{Results}

The result of the analysis is presented as themes and quotations from the focus group interviews. The themes are as follows:

- Participants' own goals as a common interdisciplinary platform.

- A positive professional community.

- Learning from each other's skills and competencies.

- New roles and joint efforts - but profession-specific competencies.

The quotes are written within double quotes. The following text describes how the rehabilitation teams experienced the interdisciplinary collaboration when assisting the participants in reablement.

\section{Participants' own goals as a common interdisciplinary platform}

From a situation of absence of interdisciplinary collaboration, where the professionals had often defined goals on patients' behalf, the professionals were moved into a context where the participants in reablement were stimulated to define their own goals. The team received an accurate description of each participant's precise goals and activities relevant for that participant. Thus, the goals were not defined by one of the professionals at the outset. One of the physiotherapists described this as almost "magical" because it created a new way of working with training and rehabilitation. Furthermore, she expressed the following on the importance of goals and their impact on the interdisciplinary collaboration: "It is not we who decide what to do, it is the patient's own goals and they do not distinguish between professions. If the goal is to wash the floors, all are equally involved."

Professionals often found that participants expressed goals and expectations regarding their rehabilitation - needs other than those that the professionals had thought of beforehand. Thus the participants' definition of their own goals was described as critically important for the interdisciplinary collaboration. To follow a participant's goals could mean that a nurse had to do tasks that traditionally were physical therapy, and a physiotherapist had to do tasks that traditionally were connected to nursing or other disciplines. However they all accepted this because they had focus on patients' goals and patients' participation in their own rehabilitation process. One physiotherapist said: "We are supporting each other in helping persons reach their own goals, although they may have a goal that is not so relevant for me."

\section{A positive professional community}

The establishment of an interdisciplinary team where everyone was more or less at the same hierarchical level, resulted in the professionals being "forced" to adhere to each other. The reason for an experience of good collaboration and community was therefore mainly attributed to the organization. Even if the experience of a positive community was mostly related to separate teams with close collaboration, they broadly experienced the collaboration as exciting, constructive and a promotion of well-being. Intensity of collaboration varied and some teams expressed the view that there could be insecurity and skepticism in the initial phase of establishing the team. However, conflicts between professionals were almost never mentioned by the teams, although this was requested in the focus group interviews. The positive attitude was largely linked to an experience of being a part of a community. The practical work with reablement could be challenging and complex, for instance, combining specific physical training, changing of stoma, motivating a depressed participant, medication and so on. While challenges could seem overwhelming for a single professional, colleagues in the team had important contributions based on their specific competencies. These contributions resulted in a sense of community and they discovered that situations had solutions that each of them not had seen in advance. Interdisciplinary working was, therefore, considered as enriching, which resulted in positive attitudes and experiences. Two professionals said: "Have a good experience of working in a team here, and we have cooperated well." "Together we manage it, everyone contributes, very positive."

Several professionals described that they had previously worked as a nurse, physiotherapist or occupational therapist where everyone was gathered in the same office building, but with no collaboration. They used to have their own patients, 
but this changed when they joined a team. A physiotherapist had the following comment: "In a team, it is not my patient, but he or she belongs to everyone, I am not left alone with that person."

\section{Learn from each other's skills and competencies}

Learning from each other by actively seeking each other's expertise was a central aspect, although how this was realized, depended on the number of different professionals in each team and how closely they collaborated. A professional from a close collaborating team said: “[We] seek each other's expertise when we have questions that are not within our field. We have respect for each other's professionalism, no disciplines are better than others. We wish each other well, and we want to learn from each other. I think that's pretty nice."

Instead of keeping their knowledge and expertise separate, they shared it with others in the team. Sharing had two aspects; the feeling of giving, guiding, increasing skills and knowledge to the others, and receiving advice, guidance and knowledge from the other professionals in the team. The following statement illustrates how strong the awareness of reciprocal learning was: "We take the best knowledge and experience from each other."

An example is that nurses could have a need for more information about the user's specific physical limitations, disability and training. Equally, the physiotherapist or occupational therapist could need information regarding the use of medication and changing of stoma. With regular meetings within the team, team members could guide and teach each other continuously: "We teach each other all the time. You get advice and guidance from others and it makes us better. It strengthens all, the input you get all the way."

This statement and the way the teams collaborated were largely dependent on talking and meeting each other to bring the different challenges to a multidisciplinary assessment. Thus specific situations could be discussed and team members learned from each other through the interdisciplinary discussion: "It's great having all the disciplines and we use both the professional background and experience when we are discussing the patients."

\section{New roles and joint efforts, but specific competence}

To be assembled into interdisciplinary independent teams led to some major changes in roles and responsibilities. An occupational therapist could change from a specialist function and relatively isolated role, to working closely with nurses in the home care service. Nurses described a change from a highly timed workday, to being in a situation with a quite different timetable. The physiotherapists had much experience with working individually with single physical functions, while to work in the person's own home as part of a team led to another way of working. Physiotherapists might tell about when they worked alone with patients, they were much more concerned about a knee, a foot or a part of the body, but the interdisciplinarity allowed them to be interested in the other parts, of the patient's life as well. The professionals established another way of working, from working almost alone to teamwork. One nurse said: "I haven't done interdisciplinary work before. I missed it when I worked in home care service. There were always meetings, but we never had time to go there."

Teamwork was considered as a working method that demanded professional community and interdisciplinarity, but it also involved individual qualities such as social skills. One professional remarked: "It relies on a community and a multidisciplinary approach to work in such a way, and I don't think it is suitable for anyone to work in this way. You must be able to share, you cannot own everything yourself."

Every professional did their own assessment of the participant based on their specific competence which included interdisciplinary discussions within the team and finally practical work based on everyone's efforts. An occupational therapist emphasized the following regarding each professional's contribution: "We look through different glasses. I, as an occupational therapist, could facilitate help at home, but if we had not put in place the diet, nutrition, medication and tried improving function, strength and balance - and after that train in daily activities, the result would not be the same."

In addition to physiotherapists, occupational therapists and nurses, some teams had professionals such as social worker, social educator and psychiatric nurse. The supplement of different professionals proved to be important because many participants also had psychosocial problems where these professions had important contributions. Sometimes they had skills and competence the others in the team did not know they needed. The close interdisciplinary work within the team therefore led to insight into different roles and what the various professions contributed with. By seeing the other professions' role, it was consequently possible to see one's own role.

\section{Discussion}

This study highlights that a prerequisite for interdisciplinary collaboration in reablement was that participants defined 
their own goals. Based on participants' goals the reablement teams experienced the collaboration as a positive professional community where they used and shared their own competencies with each other to support the participants in their rehabilitation process. Working closely in an interdisciplinary team resulted in new roles and new tasks, but at the same time it was important to be aware of their own roles as different professionals.

\section{Importance of goals}

The importance of shared goals in interdisciplinary collaboration is widely documented in various studies and must be seen as fundamental for all rehabilitation although the degree of contribution from participants will vary. ${ }^{13-15,19,23}$ In reablement, participants' contribution through own goals is usual and important and highlighted by Hjelle et al ${ }^{10}$ and Randström et al ${ }^{18 .}$ When participants are defining their own goals, reablement teams stand on a unifying platform that helps the team to obtain common challenges and goals. In the focus group interviews some professionals said that previously, they, as a single professional, used to define the training or other treatment when they worked individually with patients. These "traditional" approaches often resulted in a discrepancy between professional and participant thoughts regarding the focus for rehabilitation. Participant's ability to define their own goals therefore played a crucial role because it drew attention to the participant's wishes and needs, and consequently their motivation for rehabilitation. If a professional had defined the goals, the consequence might be a disagreement between the team members which consequently might lead to difficulties within the interdisciplinary collaboration. Defining their own goals is therefore not only a principle for participant' motivation, but is also important for the interdisciplinary collaboration.

\section{Complex situations require active collaboration}

Reablement is usually linked to activities of daily living where participants are often characterized as "experts in their own life". Reablement is therefore related to "training in everyday activities", more than specialized rehabilitation such as specific training procedures in relation to particular diagnosis or functional limitations. However, when professionals emphasize the need for different skills and the need to learn from each other, it may indicate that working with rehabilitation in people's own home can be complex. This is also consistent with a Cochrane review that points out that this is a special challenge in reablement. ${ }^{4}$ It means that reablement often includes many dimensions of life from purely physical efforts to physical environmental conditions, psychological and social circumstances. The home context and the special challenges the health workers in the present study had regarding rehabilitation in people's own home, therefore required an interdisciplinary approach and collaboration. This may also be a reason why these teams had good experiences with professionals such as social workers, social educators and psychiatric nurses, as is also highlighted by another study. ${ }^{17}$

When the professionals experienced that situations in different homes were unique, unpredictable and complex and no one had a simple and clear solution regarding participants' challenges, the reablement teams were in a need of close, intensive and time-consuming collaboration. It was not only training about a part of the body or assistance with personal hygiene or medication, but they needed to assist in coping with tasks that participants in reablement experienced as important for themselves. This required different competencies, different professional backgrounds and experiences that point toward interdisciplinarity and transdisciplinarity more than a multidisciplinary kind of collaboration. Even if there seem to be different types of multidisciplinary teams, multidisciplinarity means working individually, separately and possibly on different levels and with different work tasks. ${ }^{10}$ It may function, but the teams in this study had experienced that unpredictable and complex contexts highlight a need for close collaboration, interactivity, different disciplines, mutual planning and decision making, as in inter- and transdisciplinary teams. Time and meeting places were therefore highlighted as important. ${ }^{15,18,28}$ How teams are organized is therefore also a question about resources, and the future will show the extent to which local authorities are able to give reablement teams enough time and space to carry out their work in the best possible way.

\section{Atmosphere, climate and relations}

Interdisciplinary collaboration is often depicted as something negative, for example, in forms of competition between professionals. ${ }^{16}$ However, the current study finds that the staffs have positive experiences with teamwork. Positive experiences were related to several issues such as, for instance, a sense of community, innovation, support, humor and especially a sense of doing something meaningful for the participants in reablement. There are few studies regarding interdisciplinary collaboration within reablement, but a few recent studies confirm that overall the interdisciplinary 
teams have positive experiences with interdisciplinary collaboration in reablement teams. ${ }^{10,12,17,18}$ Climate is an aspect of work satisfaction and is a central factor in teamwork, and there is often a link between team climate and efficiency. ${ }^{19}$ Teams with a tight integrative team organization, as in transprofessional teamwork, often have a good climate characterized by openness, trust and team spirit which again lead to efficiency. ${ }^{19}$ Some municipalities had many employees in their operational teams, and these teams were organized independently from the home care service and had also an experience of tight collaboration within the team. These teams seemed to have the most positive perception and experiences of the interdisciplinary collaboration. They also seemed to have an effective quantitative and qualitative follow up of participants, probably because several professionals were fully dedicated to and shared a common focus on reablement. ${ }^{19}$

The results of this study show that team members broadly reported good interdisciplinary collaboration. This was related to a new organization and a shift from having worked mostly alone to working closely with other professionals. However, we have to take into consideration that many municipalities were in an establishment phase of reablement, and received a financial contribution from the project which may have contributed to greater freedom and more time besides the excitement and commitment that is created when establishing something new. Some professionals also had shared positions, such as physiotherapists who worked with individual patients in other departments and were able to compare the two settings. During the project they worked both independently and within the new teams. This may have played a role, but the participants in the focus groups explained that the reason for positive experiences were linked to establishing a team as a new organizational unit in which different professions were employed at the same level with common goals. Essentially, the experiences with positive collaboration are most likely a result of the organization where professionals are put together and work side by side linked to embracing common goals, even if establishing a new function and working in a new setting may have affected their opinions. They had changed from "working alone" to interdisciplinary working in a community of other professionals. At the same time, one can ask if a positive experience within the team is only a question about organization. Here it must be taken into consideration that interdisciplinary collaboration also depends on social competence and the willingness of each professional to work closely with other professionals and their motivation to contribute in an interdisciplinary team.
One of the most experienced teams expressed the view that working with reablement closely with different professionals is not suited to everyone.

\section{Learning and roles}

Learning aspects are referred to in other qualitative studies. ${ }^{12,18}$ Learning from each other was characterized as one of the exciting issues within the interdisciplinary collaboration. It may have something to do with confidence and trust, but when the organizational barriers are removed, access to new knowledge opens up. How teams are organized will, therefore, affect the learning aspect. The more there is close collaboration, time to communicate, a variety of disciplines, and shared planning and decision making, the more is the learning. In this close collaboration, each professional must be willing to give, receive and seek knowledge with colleagues in the team. When a professional from a closely collaborating team said: "Seek each other's expertise", the professional also said that team members were guiding each other. This is a consequence of the need to share knowledge and instruct one another when everyone does almost the same things and team-members meet challenges that are not within their field. Teams that had a wide variety of professionals, not only had a greater learning outcome. They also had a broader approach to rehabilitation that also included psychosocial aspects and the needs of participants.

"Roles" is a central theme in several studies concerning rehabilitation and interdisciplinary teamwork, because there will always be a tension regarding each professional's position and work tasks relative to the others in the team. ${ }^{13,18,19,22}$ The participants in the focus groups said that roles had actually been dramatically changed from an experience of working individually within their own profession, to working closely with other professionals. Potential conflicts, divergences and discussions around roles were one of the questions in the focus group interviews, but this did not become a central theme. Focus group participants indicated that there had been a discussion in the start phase, but even if much of the work had become common, they still had a perception that various professionals had different roles. Consequently, they were not in a situation where they felt they had lost their professional integrity, but they had a perception of having a more general role because they had to share tasks with others in the team.

Similarly, learning aspects, roles are influenced by the way the collaboration is organized. A key aspect is how teams are affiliated to the home care service. One type of team is the integrated multidisciplinary team where largely physiotherapists and occupational therapists have a guiding 
role for employees in the home care service who mainly perform the daily training. ${ }^{12}$ This must inevitably have consequences for collaboration, learning and roles in relation to teams working separately from the home care service with several professionals. Although some teams in this study intended to cooperate closely with home care service, collaboration at the time the focus group interviews were conducted generally can be characterized as interdisciplinary where roles were fairly specialized and everyone expected to interact. It seems to be a gradual transition between interdisciplinary and transdisciplinary teams, but some teams in this study operated independently and had a high degree of close collaboration in the direction of what one might call transdisciplinary collaboration. This means that roles were specialized and the professionals used their own specific competence, but at the same time everyone carried out the same tasks and there was a joint agreement over what to do. In this close collaboration they became aware of the other's knowledge and role, and consequently it was possible to see one's own role and knowledge.

\section{Methodological considerations}

Focus groups may reveal attitudes, experiences and perceptions by focusing on a given theme. ${ }^{26}$ The theme in the present study was interdisciplinary collaboration in reablement teams and team members were requested to share their experiences. Since this was a project, it is conceivable that the municipalities would promote their activities in a positive way, especially when leaders were present in some of the focus groups because they were a part of the team. This may have affected the group discussion in some focus groups. Differences in organization and degrees of experience with reablement are another factor that may have affected the result of the study considering that teams organized separately from the home care service had the most comprehensive experiences with collaboration. Some of these teams had also worked over a longer period than teams who aimed to work closely with the home care service, a process which would require more time. At the same time, it can be an advantage to investigate many teams with different organization, experience and maturity. Nevertheless results of this study should be viewed in light of the fact that interdisciplinary collaboration may have changed over time, especially in municipalities which aim to working closely with and integrated into the home care service. Individual interviews, in addition to observation methodology over time, would probably have provided more complementary results.

\section{Conclusion}

This study shows that interdisciplinary collaboration in reablement depends on participants in reablement having the opportunity to define their own rehabilitation goals, and on these goals functioning as a common professional platform for the interdisciplinary collaboration. If a professional defines participants' goals, there might be a disagreement between the team - members with consequences for collaboration within the team, and collaboration between the team and participants in reablement. The situation for participants in reablement is often complex, involving assessments from different professionals and close interdisciplinary collaboration. This study shows that several and varied professionals working on the same level relatively independently and separately from the home care service, are factors that will contribute to interdisciplinary collaboration. Organization of team work, including how teams are affiliated to the home care service, seems to be a key aspect for interdisciplinary collaboration in reablement. The more there is close collaboration, time to communicate, variety of disciplines, shared planning and decision making, the more there is learning, and satisfaction with the climate and atmosphere within the team. A close collaboration between several professionals entails also a broader approach which involves psychosocial aspects of participant's situation and rehabilitation - needs. Interdisciplinary and transdisciplinary collaboration is the most appropriate way to collaborate in reablement, but it causes major changes in roles from working almost alone within one's own discipline to extensive collaboration across professionals. This way of working also requires social competencies and willingness to work closely with others, but broadly team members experience team work as exciting and they perceive the relationship between team-members as positive. A close collaboration with exchanging of skills and competencies depends on each professional being conscious on their own role, special skills and competence. On the other hand they have a more general role and must be willing to take a broader range of tasks than when working more independently. Further research is needed to get more knowledge and understanding regarding how different ways of organizing of reablement affect interdisciplinary collaboration.

\section{Acknowledgments}

We would like to express our gratitude to the professionals who contributed to this study. We would also like to thank the Norwegian Directorate of Health who commissioned the nationwide study from which this qualitative study derives. 


\section{Disclosure}

The authors report no conflicts of interest in this work.

\section{References}

1. Parvaneh R, Glendinning C. Organization and delivery of home care re-ablement: what makes a difference? Health Soc Care Community. 2011;19(5):495-503.

2. King A, Parsons M, Robinson E, Jörgensen D. Assessing the impact of a restorative home care service in New Zealand: a cluster randomised controlled trial. Health Soc Care Community. 2012;20(4):365-374.

3. Tuntland H, Aaslund M K, Espehaug B, Førland O, Kjeken I. Reablement in community-dwelling older adults: a randomised controlled trial. BMC Geriatr. 2015;15:145.

4. Cochrane A, Furlong M, McGilloway S, Molloy DW, Stevenson M, Donnelly M. Time-limited home-care reablement services for maintaining and improving the functional independence of older adults. Cochrane Database Syst Rev. 2016;10:CD010825.

5. Whitehead P, Worthington E, Parry R, Walker M, Drummond A. Interventions to reduce dependency in personal activities of daily living in community dwelling adults who use homecare services: a systematic review. Clin Rehabil. 2015;29(11):1064-1076.

6. Tessier A, Beaulieu M-D, Mcginn CA, Latulippe R. Effectiveness of reablement: a systematic review. Healthc Policy. 2016;11(4):49-59.

7. Legg L, Gladman J, Drummond A, Davidson A. A systematic review of the evidence on home care reablement services. Clin Rehabil. 2016;30(8):741-749.

8. Hjelle KM, Tuntland $\mathrm{H}$, Førland $\mathrm{O}$, Alvsvåg $\mathrm{H}$. Driving forces for home-based reablement; a qualitative study of older adults' experiences. Health Soc Care Community. Epub 2016 Jan 24.

9. Wilde A, Glendinning C. "If they're helping me then how can I be independent?" The perceptions and experience of users of home-care reablement services. Health Soc Care Community. 2012;20(6):583-590.

10. Hjelle KM, Skutle O, Førland O, Alvsvåg H. The reablement team's voice: a qualitative study of how an integrated multidisciplinary team experiences participation in reablement. J Multidiscip Healthc. 2016;9:575-585.

11. Steihaug S, Lippestad JW, Isaksen H, Werner A. Development of a model for organisation of and cooperation on home-based rehabilitation - an action research project. Disabil Rehabil. 2014;36(7):608-616.

12. Moe A, Brataas HV. Interdisciplinary collaboration experiences in creating an everyday rehabilitation model: a pilot study. $J$ Multidiscip Healthc. 2016;9:173-182.
13. Sargeant J, Loney E, Murphy G. Effective interprofessional teams: "contact is not enough" to build a team. J Contin Educ Health Prof. 2008;28(4):228-234.

14. Johansson G, Eklund K, Gosman-Hedström G. Multidisciplinary team, working with elderly persons living in the community: a systematic literature review. Scand J Occup Ther. 2010;17(2):101-116.

15. White M, Gutierrez A, McLaughlin C, et al. A pilot for understanding interdisciplinary teams in rehabilitation practice. Rehabil Nurs. 2013;38(3):142-152.

16. Hall P. Interprofessional teamwork: professional cultures as barriers. J Interprof Care. 2005;19(Suppl 1):188-196.

17. Liaaen JMA. Professional careers' experience of working with reablement [master]. Trondheim: Fakultet for helse- og sosialvitenskap, Høgskolen i Sør-Trøndelag; 2015.

18. Randström K, Wengler Y, Asplund K, Svedlund M. Working with 'hands-off' support: a qualitative study of multidisciplinary teams' experiences of home rehabilitation for older people. Int J Older People Nurs. 2014;9(1):25-33.

19. Thylefors I, Persson O, Hellström D. Team types, perceived efficiency and team climate in Swedish cross-professional teamwork. J Interprof Care. 2005;19(2):102-114.

20. Donohue K, Hoevenaars R, McEachern J, Zeman E, Mehta S. Homebased multidisciplinary rehabilitation following hip fracture surgery: what is the evidence? Rehabil Res Practice. 2013;2013:875968.

21. Duner A. Care planning and decision-making in teams in Swedish elderly care: a study of interprofessional collaboration and professional boundaries. J Interprof Care. 2013;27(3):246-253.

22. Pellatt G. Perceptions of interprofessional roles within the spinal cord injury rehabilitation team including commentary by Lingard L. Int $J$ Ther Rehabil. 2005;12(4):143-150.

23. Langeland E, Førland O, Aas E, et al. Models for reablement - a followup evaluation in Norwegian municipalities. Norwegian Directorate of Health. Centre for Care Research Western Norway. Report number 6/2016. (Report on Norwegian). 2016.

24. Patton MQ. Qualitative Research \& Evaluation Methods. 3rd ed. Thousand Oaks, CA: Sage Publications; 2002.

25. Krueger A, Casey M. Focus groups. A practical guide for applied research. 3rd ed. Thousand Oaks, CA: Sage Publications; 2000.

26. Alvesson M, Skøldberg M. Reflexive Methodology. New Vistas for Qualitative Research. London: Sage; 2000.

27. San Martín-Rodríguez L, Beaulieu M, D'Amour D, Ferrada-Videla M. The determinants of successful collaboration: a review of theoretical and empirical studies. J Interprof Care. 2005;19(Suppl 1):132-147.
Journal of Multidisciplinary Healthcare

\section{Publish your work in this journal}

The Journal of Multidisciplinary Healthcare is an international, peerreviewed open-access journal that aims to represent and publish research in healthcare areas delivered by practitioners of different disciplines. This includes studies and reviews conducted by multidisciplinary teams as well as research which evaluates the results or conduct of such teams or health

\section{Dovepress}

care processes in general. The journal covers a very wide range of areas and welcomes submissions from practitioners at all levels, from all over the world The manuscript management system is completely online and includes a very quick and fair peer-review system. Visit http://www.dovepress.com/ testimonials.php to read real quotes from published authors. 